\title{
Documentos legales y oficiales de la frontera del nordeste del reino de Granada (siglo XV)
}

\author{
Legal and official documents in the northeast border \\ of the Kingdom of Granada (15th century)
}

\author{
María ARCAS CAMPOY \\ Universidad de La Laguna \\ marcas@ull.es
}

Recibido: 23 de noviembre de 2014

Aceptado: 3 de mayo de 2015

\begin{abstract}
RESUMEN
En los archivos Municipal de Lorca (Murcia) e Histórico de Orihuela (Alicante) se conservan una serie de documentos que contienen información sobre algunos aspectos de la vida de la frontera del nordeste del Reino de Granada en el siglo XV. Estos documentos son de dos tipos: legales (escrituras notariales y judiciales) y oficiales (cartas bermejas y una parte de la correspondencia institucional generada por cuestiones políticas). Presentamos aquí referencias y extractos de estos documentos que constituyen una estimable contribución al conocimiento de la vida social, política y económica del último siglo del reino nazarí.
\end{abstract}

Palabras clave: Reino nazarí de Granada: Frontera del nordeste, documentos legales, cartas bermejas, correspondencia oficial.

\begin{abstract}
A number of documents containing information about day life in the northeast border of the Kingdom of Granada (15th century) are preserved in the Archivo Municipal de Lorca (Murcia) and in the Archivo Histórico de Orihuela (Alicante). These documents are of two types: legal documents (notarial and judiciary documents) and official documents (cartas bermejas and institutional correspondence produced by political conflicts). This paper presents references and extracts of those legal and official documents to illustrate the social, politic and economic life of individuals in the last century of the Nasrid Kingdom of Granada.

Keywords: Nasrid Kingdom of Granada: the northeast border, Legal documents, "cartas bermejas", institutional correspondence.
\end{abstract}

SUMARIO: 1. Documentos legales, 2. Documentos oficiales, 2.1 Cartas Bermejas, 2.2 Correspondecia oficial, 3. Conclusiones 
La vida en la frontera nordeste nazarí durante el siglo XV generó, además de numerosos documentos de parecidas características a los del resto del reino de Granada, otros tipos de escritos referentes a cuestiones específicas de este territorio' ${ }^{1}$. La ciudad de Vera era la principal población limítrofe de esta frontera terrestre y marítima, una zona alejada de la capital, en permanente alerta frente al cercano enemigo y con una difícil situación social, política y económica. No obstante, la actividad de los habitantes de sus ciudades y aldeas así como la de sus autoridades era vibrante, rica e incesante como ponen de manifiesto varias fuentes y estudios sobre el tema. Y esta actividad, en parte inherente a la vida de frontera y en parte originada por los acontecimientos, quedó plasmada en documentos de diferentes tipos cuya existencia se conoce principalmente a través de referencias y en menor medida de los escasos originales conservados, que permiten, al menos, una aproximación a la naturaleza y los contenidos de los mismos.

En los Archivos Municipal de Lorca e Histórico de Orihuela se conservan varios documentos cuya información permite distinguir dos tipos de escritos: legales y oficiales.

\section{DOCUMENTOS LEGALES}

Bajo esta denominación se incluyen diversos tipos de escrituras notariales y judiciales redactadas a instancias de particulares o de representantes de la administración de la justicia, de cuya existencia, por lo que se sabe hasta el momento, solo han llegado referencias recogidas en otros documentos. No obstante, dichas referencias proporcionan importantes datos como veremos seguidamente.

Gran parte de la información al respecto procede del Archivo Municipal de Lorca, donde se encuentra abundante documentación generada por el pleito entre Lorca y Vera por la delimitación de sus términos, un largo pleito que, iniciado en 1511, se prolongó en distintas fases y revisiones hasta finales del siglo XVIII sin llegar a un final totalmente satisfactorio para las partes. La casi totalidad de las referencias sobre escritos de esta zona fronteriza se encuentra en las Probanzas ${ }^{2}$ (Sala II, 217 bis 3 ), una rica fuente de información que contiene las declaraciones de los testigos de la primera fase de dicho pleito, desde 1511 hasta 1559. Las Probanzas, una de las principales piezas utilizadas en el largo y arduo pleito, ponen de manifiesto las posturas enfrentadas de las partes en litigio a través de las declaraciones de los correspondientes testigos respecto a sus derechos jurisdiccionales sobre Huércal y Overa, remontándose a "tiempo de moros", pues mientras Lorca alegaba que ambas villas fueron independientes y gobernadas por personas nombradas directamente por el rey de Granada, por el contrario la parte de Vera sostenía que siempre estu-

\footnotetext{
${ }^{1}$ Este trabajo está integrado en el proyecto de investigación: "Documentos de la Granada nazarí y mudéjar: estudio de las colecciones (derecho, economía y sociedad)". Ministerio de Ciencia e Innovación FFI2012-37775.

${ }^{2}$ Consta de dos volúmenes: Parte de Vera y Parte de Lorca (En adelante cito PV= parte de Vera y $\mathrm{PL}=$ parte de Lorca)
} 
vieron a todos los efectos bajo su autoridad. Es conveniente recordar que ambas villas, conquistadas en 1488 y cedidas a Lorca por una merced de los Leyes Católicos, permanecieron bajo su jurisdicción hasta la creación del municipio independiente de Huércal-Overa en $1648^{3}$.

Los testigos de las dos partes del mencionado pleito, la mayoría antiguos súbditos nazaríes de avanzada edad y que conocieron de primera mano u oyeron contar a sus mayores multitud de sucesos y anécdotas, hacen referencia entre otros asuntos a los nombramientos de los cadíes y a sus funciones en esta parte de la frontera naza$\mathrm{ri}^{4}$. Como es de suponer las declaraciones de los testigos de las partes en litigio son totalmente opuestas ya que los de Lorca alegaban que los cadíes de Huércal y Overa eran nombrados directamente por el sultán granadino, mientras que los de la parte contraria afirmaban que ambas villas carecían de cadí y por ello se veían obligados a acudir al magistrado de Vera. En las declaraciones de los testigos, recogidas en los dos volúmenes de las citadas Probanzas, se encuentran numerosas referencias a diferentes tipos de escrituras redactadas en las últimas décadas del siglo $\mathrm{XV}$, que desgraciadamente no se conservan.

En 1550 y 1558 la parte de Lorca pregunta a varios testigos sobre las actividades y funciones de los cadíes itinerantes por el reino de Granada. Entre las numerosas respuestas que aluden a esta cuestión destacan las siguientes:

El d(ic)ho cadi haçia partiçiones de bienes y otros autos a estos tocantes (Juan El Cantori, año 1550, PL, fol. 164v); los cadis eran nombrados por el rrei moro o por el cadi general que residía en Granada [...] hante los d(ich)os cadis los beçinos del di(ch)o reino de Granada haçian cartas de almahar, que son las cartas de docte de los que se casavan y que los d(ich)os cadis haçian particiones de las haçiendas que dexavan los moros que se morian (Garçia El Forruchel, año 1558, PL, fol. 234r); avia cadis que heran puestos e nombrados por el rrei moro de Granada, los quales di(ch)os cadis este testigo vio que andavan usando su oficio porque haçian cartas de docte e partiçiones de los que se morían (Gonçalo Hernandez Axir, año 1558, PL, fol. 234v); por el rreino de Granada andavan cadis haçiendo cartas de docte y particiones porque esto lo haçian los d(ich)os cadis por mandado del rei como personas savias que heran (Pedro de Málaga, año 1559, PL, fol. 234v); avia cadis que heran puestos por el rrei moro o por su cadi general que estaba e rresidia en Granada y que estos cadis, dixo este $\mathrm{t}(\mathrm{e}) \mathrm{s}(\mathrm{tig}) \mathrm{o}$, que los $\mathrm{v}($ ecin)os del dicho rreino de Granada haçian sus cartas de almahar con ellos e haçian partiçiones (Miguel Fotox Aluani, año 1558, PL, fol. 234v-235r).

\footnotetext{
${ }^{3}$ Sobre la historia de estas villas véase, VEAS ARTESEROS, Francisco, "La cesión de Huércal y Overa a Lorca en 1488", en Roel 4 (1983) 63-80; y JIMÉNEZ ALCÁZAR, Juan Francisco, "Huercal y Overa. Entre dos reinos (1488-1520)", en Actas del II congreso de Historia de Andalucía. Córdoba, 1991, (1991) 393-399, y Huércal y Overa: De enclaves nazaries a villa cristianas (1244-1571), Huércal-Overa 1996.

${ }^{4}$ Véase, ARCAS CAMPOY, María, "Noticias sobre el cadiazgo en los últimos años del reino nazarí: la frontera entre Murcia y Granada", en Revista del Centro de Estudios Históricos de Granada y su Reino 6, $2^{\mathrm{a}}$ época (1992) 203-210.
} 
Por la parte de Vera las preguntas y respuestas, siempre encaminadas a demostrar la pertenencia de Huércal y Overa a la ciudad de Vera, también aluden a la redacción de documentos legales. En 1550 y 1559 varios testigos son llamados a declarar, entre sus testimonios destacan los siguientes:

En la dicha ciudad de Vera estava e rresidia un cadi que conosçia de los pleytos çiviles que se tratavan en la dicha ciudad de Vera el qual dicho cadi este testigo bio que conoçia ansimismo de todos los pleitos çiviles que se seguían y tratavan entre los veçinos de los dichos lugares de Huercal y Overa y ante el dicho cadi otorgaban sus escripturas los dichos vecinos de los dichos lugares ansi cartas de docte como partiçiones y otras cualesquier escripturas (Diego El Chilichi, año 1550, PV, fol. 201v202r); y cuando avian de hazer algunas escripturas y cartas de docte en los dichos lugares de Guercal y Overa el dicho su padre era llamado para ello [...] y que para conocer de los dichos pleitos y façer las dichas escripturas y para casar e descassar y para todo lo demás que tocasse a pleitos civiles su padre deste testigo tenia provission del rrey moro que se llamava Muley Abulhazen; yba con el dicho su padre como cadi de los dichos lugares a Guercal y Overa a haçer partiçiones e otras escripturas (Gonzalo Yaçid, año 1550, PV, fol. 203v, 204v, 212r-212v); quando los vecinos de los dichos lugares habían de haçer algunas escripturas de docte y partiçiones y qualesquier escriptura que las tales escripturas las yban a façer a este cadi que estaba e rresidia en la dicha ciudad de Vera (Luis Quexuel, año 1550, PV, fol. 218v); cuando avian de façer algunas escripturas de docte y partiçiones y otras qualesquier escripturas que ynbiavan a llamar a un escribano que estaba en la dicha ciudad de Vera que se llamava El Bejari el qual yba a hazer las dichas escripturas con poder del cadi que estaba en la dicha ciudad de Vera (Alonso El Haquen, año 1550, PV, fol. 220r); cualesquier escritura que se haçia la haçia el alfaqui que llaman clérigo el qual alfaqui ponía la justicia de Vera (Pedro de Mercado, año 1559, PV, fol. 333v).

Una veintena más de testigos se refiere a estos documentos utilizando expresiones y términos como:

escripturas de cartas de docte y particiones y otras qualesquier escripturas; escripturas de cartas de docte y partiçiones y otras qualesquier escripturas y autos; "escripturas, cartas de docte y partiçion; cartas de almahar que son cartas de docte de los que se casavan; carta de docte e casamiento; escripturas de docte e conciertos de casamientos o otros cualquier autos de justicia; escripturas de casamientos e otras cosas; escripturas de bentas; escriptura de compra; autos e escripturas judiçiales; escripturas, cartas de docte, como partiçiones y otras cualesquier escripturas.

No hay duda de que los escritos mencionados por los testigos de ambas partes del Pleito se refieren principalmente a los distintos tipos de actas notariales que se redactaban en el reino de Granada siguiendo probablemente, por proximidad espacial y temporal, los modelos contenidos en el 'Iqd al-munazzam li-l-ḥkkām del granadino Ibn Salmūn (m. 767/1365), lo que no descarta que también tuvieran vigencia

${ }^{5}$ Se trata del sultán Abū 1-Hasan ${ }^{\mathrm{c}}$ Alī $\left(1^{\circ}\right.$ reinado: 869/1464/887/1482; $2^{\circ}$ reinado: $888 / 1483 / 89 / 1485)$. 
formularios notariales andalusíes de siglos anteriores como el Kitāb al-wațầ 'iq wal-siŷillāt de Ibn al- Ațtār (399/1009), al-Muqni' fì 'ilm al-šurūt de Ibn Mugīt (495-1066) y al-Maqșad al-mahmūd fí taljịs al- uqūd de al-Ŷazīrī (m. 585/1189), entre otros. No obstante, las expresiones utilizadas en algún caso -"autos e escripturas judiciales" y "otras qualesquier escripturas y autos"- permiten apuntar a otro tipo de escritos relacionados con diferentes trámites procesales, como la citación $\left(r a f^{\prime}\right)$, los alegatos (maqālāt) y las sentencias ( $\left.a h k \bar{a} m\right)$, que quedaban registradas en un dīwān judicial.

Pese a que la mayoría de las referencias a los documentos legales es de carácter general e inespecífico, como se advierte en las expresiones "escripturas", "qualesquier escripturas" o "escripturas judiciales", en algunos casos constan claramente los asuntos objeto de las escrituras entre los cuales figuran matrimonios, repudios, particiones y compraventas. Se trata, pues, de actas notariales muy representativas y frecuentes de la vida cotidiana de los habitantes de Vera y de otras poblaciones del entorno fronterizo. Las escrituras de matrimonio (nikāh) son las más citadas por los testigos, especialmente las de dote (mahr / șadāq) ${ }^{6}$, como se desprende de frases como "cartas de almahar, que son las cartas de docte de los que se casavan; cartas de docte; cartas de almahar; escripturas de docte; escripturas de cartas de docte; carta de docte e casamiento; escripturas de casamientos". También hay una alusión a documentos de repudio en el testimonio de Gonzalo Yaçid, (año 1550, PV, fol. 204v) quien afirma que su padre, el cadí de Vera Abraen Aben Yaçid', tenía el nombramiento del sultán granadino para "façer las dichas escripturas y para casar e descassar".

Por último, aunque en menor número, también hay referencias a actas de partición de bienes (qisma) - "partiçiones de los que se morían; partiçiones de las haçiendas que dexavan los moros que se morian; partiçiones; escripturas de partiçion-, de compra - "escriptura de compra"-y de venta - "escripturas de bentas"-.

La persona encargada de redactar los citados documentos, según la mayoría de los testigos, era el cadí. Tan solo en dos casos son mencionados un alfaquí, que al parecer también ejercía el cadiazgo en Vera, y un escribano. De estos datos se desprende que, al menos en este espacio y tiempo, solía ser el propio cadí quien redactaba las actas de los asuntos solicitados, lo que por otra parte no resulta extraño ya que esta actividad podía ser realizada no solo por los notarios sino también

${ }^{6}$ CARMONA, Alfonso, "Aportación al estudio del contrato matrimonial en el occidente islámico medieval" en Miscellanea Arabica et Islamica, Orientalia Lovaniensia Analecta 52, F. De Jong (ed.) (1993), p. 54, indica que este término designa "en documentos magrebíes, especialmente tunecinos, la parte del acidaque cuyo pago queda aplazado". ZOMEÑO, Amalia, Dote y matrimonio en al-Andalus y el norte de África. Estudio sobre la jurisprudencia islámica medieval, Madrid: CSIC, 2000223 , explica que en al-Andalus la dote aplazada se llamaba kāli' mientras que en el resto del occidente islámico se utilizaba el término mahr.

${ }^{7}$ Sobre este cadí, véase ARCAS CAMPOY, María, "Habraen Aben Yaçid, cadí de Vera en los últimos años del reino nazari", en Boletín de la Asociación Española de Orientalistas XLI (2005) 255-265. 
por los magistrados 8 . Sin embargo la mención de un escribano, llamado El Bejari, sí parece corresponder a la figura de un notario propiamente dicho (wattāq, muwattiq, mursim, kätib al-wata $\left.{ }^{\prime} i q\right)^{9}$ cuyo principal cometido era redactar las actas y dar fe de sus contenidos. Asimismo corrobora esta afirmación una referencia a un escrito de dote -"una carta que estava en pergamino en aravigo la qual declaró Juan Martinez alfaqui"- que figura en un documento de partición de bienes de Adrián de Huércal, del año 1533, conservado en el Archivo Municipal de Lorca ${ }^{10}$.

Dicha referencia pone de manifiesto, entre otras cosas, que se trata de un acta redactada en los últimos años del reino nazarí o en la etapa mudéjar que mantenía su validez y vigencia en el sistema jurídico castellano de la época y que el intérprete, y tal vez redactor de la misma, llevaba unido a su nombre el título de alfaquí, como persona versada en el derecho islámico ${ }^{11}$.

\section{DOCUMENTOS OFICIALES}

En este tipo de documentos se incluyen los escritos y la correspondencia de carácter oficial referentes a la zona fronteriza nordoriental del reino de Granada. Algunos de estos documentos, que afortunadamente se conservan, como es el caso de varias cartas bermejas y parte de la correspondencia mantenida entre las autoridades de Vera, Lorca y Orihuela sobre un asunto de prisioneros, constituyen una rica y valiosa fuente de información sobre nombramientos de cargos institucionales, provisiones reales, polémicas y vías de resolución de conflictos, etc.

\footnotetext{
${ }^{8}$ Según LÓPEZ ORTIZ, José, "La jurisprudencia y el estilo de los tribunales musulmanes de España", en Anuario de Historia del Derecho Español IX (1932) 246-247, los propios cadíes redactaban actas. Asimismo CARMONA, Alfonso, "Aportación al estudio", 54, indica que las actas podían ser redactadas por un notario o un magistrado.

${ }^{9}$ Sobre los notarios y su origen como testigos instrumentales, véase ARIÉ, Rachel, L'Espagne musulmane au temps des naṣrides (1232-1492), París (reimpresión) 1990 287-292; e IBN AL- ${ }^{\mathrm{C}} \mathrm{ATT} \overline{\mathrm{A} R}$, Formulario notarial y judicial andalusí, Estudio y traducción de P. Chalmeta y M. Marugán, Madrid 2000 pp. 9-14 del estudio. Sobre la doble función del notario como redactor de documentos y testigo instrumental hay que señalar, los trabajos de ZOMEÑO, Amalia, "Notaries and their formulas. The legacies from the University Library of Granada", en From al-Andalus to Khurasan. Documents from the Medieval Muslim Word, M. Sijpesteijn, L. Sundelin, S. Toralla Tovar y A. Zomeño (eds.), Leiden 2007 59-77; y "Del escritorio al tribunal. Estudio de los documentos notariales en la Granada nazarí", en Graphîon. Códices, manuscritos e imágenes. Estudios Filológicos e Históricos, J. P. Monferrer Sala y M. Aldón (eds.), Córdoba 2003 75-98 (pp. 83-84).

${ }^{10}$ Este documento (Archivo Municipal de Lorca, Protocolo $\mathrm{n}^{\mathrm{o}}$ 8, junto a otras escrituras redactadas entre los años 1512 y 1591) ha sido editado y estudiado por ARCAS CAMPOY, María, "Partición de bienes del morisco Adrián de Huércal (año 1536)", en Miscelánea de Estudios Árabes y Hebraicos 59 (2010) 3-20.

${ }^{11}$ IBN AL-'ATTTĀR, Formulario notarial, pp. 10-11 del estudio: entre los requisitos de los notarios figuran la buena letra, el dominio del árabe, el dominio del derecho, el conocimiento del cálculo para las herencias y el conocimiento de la técnica notarial.
} 


\subsection{CARTAS BERMEJAS}

En el Archivo Municipal de Lorca se conserva una pieza incorporada en 1551 al Pleito entre Lorca y Vera (Sala II, 217 bis 2), que contiene la versión romanceada de varias cartas bermejas. Por encargo de Lorca y para demostrar que las villas de Huércal y Overa nunca dependieron de la autoridad de Vera en "tiempo de moros", el escribano romanceador, Juan Rodríguez, realizó la versión al castellano de unas cartas en árabe -lamentablemente perdidas- que se hallaban en posesión del concejo lorquino. Son un total de nueve cédulas o cartas reales ${ }^{12}$ las que Juan Rodríguez romanceó en la villa de la Calahorra, del marquesado del Cenete. Así consta en el documento ${ }^{13}$ :

Copia de las Cartas Bermejas escriptas en aravigo que los reyes moros de Granada enviaron a la villa de Huercal y a otros ofiçiales della, que por parte de la ciudad de Lorca se presentaron en el pleito que trata con la çibdad de Bera sobre el término y canpo de la dicha Huercal. Las quales romaço y traduxo del aravigo Juan Reodriguez escribano romanceador de las escripturas en la çibdad y reyno de Granada por su magestad aviendo sido requerido para ello por Luis Perez reçeptor a pedimento de la çibdad de Lorca en tres días del mes de março de 1551 años. Las romanço y traduxo cada una de por si en los días que de yuso serán declarados y las dio juradas y signadas y son del thenor siguiente.

Dichas cartas, denominadas bermejas por ser de color rojizo el papel de los escritos oficiales de los monarcas nazaríes ${ }^{14}$, fueron remitidas entre los años 1409 y 1488 a personas con cargos oficiales y a vecinos de la villa y fortaleza de Huércal, notificándoles nombramientos y concesiones de derechos a percibir impuestos, entre otros asuntos ${ }^{15}$, como puede apreciarse en los resúmenes de sus contenidos expuestos a continuación por orden cronológico ${ }^{16}$, que no es el seguido en el documento:

${ }^{12}$ Estas cartas ya han sido estudiadas por ESPINAR MORENO, Manuel y GRIMA CERVANTES, Juan, "Estudio de algunas cartas de los reyes nazaríes dirigidas a los habitantes de Huércal (14091488)", en Revista del Centro de Estudios Históricos de Granada y su Reino, 2 (1988) $2^{\mathrm{a}}$ época 39-57, y por FONTELA BALLESTA, Salvador, "Nueve cédulas reales de los últimos sultanes nazaríes", en Revista Cultural Huércal-Overa 8 (1990) 35-41, "Nueve cédulas reales de los últimos sultanes nazaríes (Primera parte)", en Boletín de la Asociación Española de Orientalistas (=BAEO) XXVIII (1992) 195-212 y "Nueve cédulas reales de los últimos sultanes nazaríes (Segunda parte)", en BAEO XXIX (1993) 17-30.

${ }^{13}$ ESPINAR MORENO, Manuel y GRIMA CERVANTES, "Estudio de algunas cartas", 57, ofrecen este fragmento en el documento $\mathrm{n}^{\circ} 10$ del apéndice documental.

${ }^{14}$ ARIÉ, Rachel, Historia de España musulmana (siglos VIII-XV), en M. Tuñón de Lara (dir.), Historia de España, III, Barcelona, 198268.

${ }^{15}$ Véase la transcripción de la nueves cartas en ESPINAR MORENO, Manuel y GRIMA CERVANTES, Juan, "Estudio de algunas cartas", 49-57; y FONTELA BALLESTA, Salvador, "Nueve cédulas reales", en BAEO XXIX 22-29.

${ }^{16}$ Sigo la cronología y nombres de los sultanes nazaríes ofrecida por VIDAL CASTRO, Francisco, "Historia política" en $\mathrm{M}^{\mathrm{a}}$ Jesús Viguera (ed.), El reino nazarí de Granada (1232-1492). Política, instituciones, espacio y economía - Historia de España. Menéndez Pidal, dirigida por José $\mathrm{M}^{\mathrm{a}}$ Jover Zamora, Madrid 2000, tomo VIII-3 151-248. 
1. Cédula del año 812/1409 firmada por el sultán Yuçaf, es decir Yūsuf III (810/1408-820/1417), en la que se ordena al almojarife de Purchena que entregue a los vecinos de Huércal "lo que an de aver por los libramientos, entregamiento cumplido sin que se pueda tocar cosa alguna dello para siempre".

2. Cédula del año 841/1438 firmada por el sultán Mahamad, que por la fecha se deduce que se trata de Muhammad IX El Zurdo (tercer reinado 836/1432-849/1445). La carta va dirigida a "Abraen, hijo de Yuçaf el Baguiz", otorgándole el nombramiento "de caudillo y alguaçil en su lugar y fortaleza de Huercal, cargo que le honrra por labor de sus obras".

3. Cédula del año 853/1449 ${ }^{17}$ firmada por el sultán Mahamad, cuyo nombre puede corresponder a Muḥammad IX el Zurdo (cuarto reinado 822/1447857/1453) o tal vez a Muhammad X El Chiquito (primer reinado 857/1453858/1454), en la que se nombra a Yuçaf, hijo de Mahamad el Bagued, veedor de "armas y lanzas y de otras cosas que ay en la Calahorra y la fortaleza de Huercal."

4. Cédula del año 857/1453 en la que se conceden los diezmos de los parajes de Teresa y Cabrera al "aguazil y biejos y gente común vecinos de Huercal". No consta el nombre del sultán pero por la fecha podría ser Muhammad X el Chiquito (primer reinado 857/1453-858/1454).

5. Cédula del año 858/1454 en la que se ratifica la entrega de los diezmos de Teresa y Cabrera a la villa de Huércal. Tampoco aparece en este escrito el nombre del sultán pero, por el contenido de la carta ratificando lo ordenado en la anterior, lo más probable es que también se trate de Muhammad X el Chiquito (primer reinado 857/1453-858/1454) ${ }^{18}$.

6. Cédula del año 859/1455 firmada por el sultán Mahamad, que probablemente se refiera también a Muhammad X el Chiquito (segundo reinado, 859/1455), con el nombramiento de Galib Aben Abuguaqui como alcaide de Huércal "por ser alcaide ensalçado guerreador noble acatado a mi".

7. Cédula del año 873/1468 firmada por el sultán Ali, que corresponde a Abū 1-Ḥasan ${ }^{c}$ Alī (primer reinado 869/1464-887/1482), Muley Hacén, en la

\footnotetext{
${ }^{17}$ En el documento figura el año 1489 como el equivalente al 893 de la hégira, sin duda debido a un descuido del copista, como advierte FONTENLA BALLESTA, Salvador, "Nueve cédulas reales", en BAEO XXVIII, 199, indicando la fecha correcta de 1449. Sin embargo, ESPINAR MORENO, Manuel y GRIMA CERVANTES, "Estudio de algunas cartas", 56-57, afirman que la carta está firmada por Muhammad XII (en realidad Muhammad XI, Boabdil), en 893/1488, lo que no se corresponde con las fechas que aparecen en el documento.

${ }^{18}$ FONTENLA BALLESTA, Salvador, "Nueve cédulas reales" de los últimos sultanes nazaríes", en $B A E O$ XXIX, 17, sugiere que se trata del emir $\mathrm{Sa}^{c} \mathrm{~d}$ (primer reinado 858/1454-859/1455, segundo reinado 1455-1462, tercer reinado 1463-1464).
} 
que ordena que los derechos y magranes" del lugar de Ohanez "se repartan y hagan según que se a fecho por cuanto son para Huercal".

8. Cédula del año 890/1485 firmada por el sultán Mahamad, que por la fecha puede identificarse con Muhammad XII El Zagal (890/1485892/1487), en la que nombra alcaide de Urracal al escudero Abdul Gani, hijo de Galib Abuguaquil, encargándole el "acompañamiento del biejo alcaide Hamo el Mozaguaque".

9. Cédula del año 891/1486 firmada por el sultán Mahamad, que por la fecha también puede referirse a Muhammad XII el Zagal (890/1485-892/1487), en la que ordena entregar a los vecinos y al alcaide de Urracal, el citado Abdul Gani, hijo de Galib Aben Abuguaquil, el sueldo de tres escuderos.

Además de estas cartas bermejas, hay referencias a otras misivas de los últimos sultanes nazaríes en las que consta el nombramiento de varias personas como alcaides de Huércal y Overa, según las declaraciones de tres testigos recogidas en las citadas Probanzas del pleito entre Lorca y Vera. Juan Chacon (PL, 1517, 67r) afirma que en la fortaleza de Huércal el alcaide "estava puesto de mano del rei de Granada y por carta del d(ic)ho rey que este testigo oyo leer que se la mostro el alcaide y hera bermejada colorada" y añade que llegó otro alcaide natural de Loja y que "trujo carta del rey de Granada para ser alcayde y la mostro a las gentes del dicho lugar en la mezquita". Otro testigo, Fernando Merin (PL, 1517, PL, 67v), indica que el alcaide de Huércal era un tal El Moratali "el cual vino por alcaide por mano del rei moro porque vido la carta del rei moro de Granada Mulei Malhaçen ${ }^{20}$ por la qual le haçia amo de la alcaidia de Guercal'. El tercer testigo, Francisco de Morales (1550 PL, fol. $128 \mathrm{v}$ ), se refiere a esta cuestión indicando que el alcaide de Huércal y Overa fue nombrado por el "rrey moro que en aquella sazon reinaba que se llamaba Mulei Acen" por una "carta bermexa".

Por último, hay que añadir algunas referencias a otras cartas firmadas por los monarcas granadinos en las que se otorgaba el perdón a los homicianos retraidos ${ }^{21}$ en las

\footnotetext{
19 "Magran" es el nombre de un impuesto sobre las propiedades rústica en tiempos de los nazaríes que, con modificaciones, también se aplicaba tras la conquista de Granada. Sobre esta cuestión véase el artículo de RUIZ PÉREZ, Ricardo, "El magran, impuesto decisivo en la progresiva señoralización del Marquesado del Cenete durante la época morisca", en Chonica Nova 14 (1984) 193-328, especialmente p. 298. El término "magran", como indica CORRIENTE, Federico, Diccionario de arabismos y voces en iberorromance, Madrid 1999, 185, las voces almagrán y magram, "cierto tributo" proceden del árabe andalusí almagrám, "tributo", y éste a su vez del clásico magram, "deuda".

${ }^{20} \mathrm{O}$ tal vez Muleim Alhacen ya que la lectura es dudosa.

${ }^{21}$ Este tema ha sido abordado por ARCAS CAMPOY, María en "Ortodoxia y pragmatismo del fiqh. Los 'homicianos' de la frontera oriental nazarí", en Orientalia Lovaniensia Analecta 86, Proceedings of the Eighteenth Congress of the Union Européenne des Arabisants et Islamisants (Lovaina, 1996), U. Vermeulen y J.M.F. van Reeth (eds.), (1998) 75-85; y "Fuentes sobre los delitos de sangre en al-Andalus: Dos ejemplos referidos a Córdoba (siglos X-XI) y la frontera oriental nazarí (siglo XV)", en Clio\&Crimen, 10 (2013) 95-109.
} 
fortalezas de Huércal y Overa. Los llamados homicianos, a los que la justicia ordinaria no podía juzgar ni castigar los delitos cometidos fuera de dichos lugares, redimían sus penas a cambio de permanecer un tiempo estipulado defendiendo en primera línea estas fortalezas fronterizas tan peligrosas como ruinosas y desabastecidas.

Algunos testigos del ya citado pleito entre Lorca y Vera mencionan haber visto o tener referencias de cartas bermejas con el perdón del sultán a los huidos de la justicia que llegaban a Huércal y Overa, entre ellos Fernando Merin (año 1517, PL, fol. $67 \mathrm{v}$ ), anteriormente citado, quien declara que "vido que avia en la d(ic)ha Huercal quatro o çinco hombres que abian muerto hombres en Vera e que estaban alli seguros porque avia una carta del rrei moro ${ }^{22}$ para que sirbiendo en Guercal çiertos años los perdonaba el rei moro".

\subsection{CORRESPONDENCIA OFICIAL}

La correspondencia oficial es otra interesante fuente de información sobre el espacio fronterizo que nos ocupa. En el Archivo Histórico de Orihuela (Libro A-30. Sobre I) se conserva parte de la correspondencia mantenida entre la ciudad de Vera y Lorca y Orihuela en los últimos años del reino nazarí motivada por el apresamiento y posterior venta de unos hombres de Vera en tiempo de paz. Entre estos documentos destaca una carta escrita en árabe y fechada en el año 879/1471, enviada por la Asamblea de autoridades (mahfil) de Vera al Consell de Orihuela en cuya gestión interviene el Concejo de Lorca ${ }^{23}$. Se trata pues de una misiva que afecta a tres reinos fronterizos entre sí: el de los Banū Nașr de Granada y los de Murcia y Valencia, integrados respectivamente a las Coronas de Castilla y Aragón.

El documento ocupa un folio con parte del texto en el margen donde, junto a obligadas frases de cortesía, consta la fecha: 11 de $r a b \imath^{c}$ al-äjir del año 879/25 de agosto de 1474. En la otra cara del folio, plegada a modo de sobre, figura como destinatario el Consell de Orihuela (al-mahfil bi-madīna Uryūla).

En la carta las autoridades de Vera, con la mediación de Lorca, reclaman, en cumplimiento del acuerdo de tregua suscrito por ambas partes, la liberación de "unos moros de Vera" que fueron cautivados en tiempo de paz y llevados a Valencia e Ibiza La reclamación, en términos correctos pero firmes y rotundos, recuerda a los de Orihuela que ya hicieron esta misma petición ocho meses atrás, dejando entrever las consecuencias de su incumplimiento en la frase: "No nos hagáis soportar más de lo que [ya] nos habéis hecho soportar". Por fortuna se

${ }^{22}$ Se trata Abū 1-Hasan ${ }^{c}$ Alī (primer reinado 869/1464-887/1482), Muley Hacén.

${ }^{23}$ Este interesante documento ha sido estudiado por MARTÍNEZ ALMIRA, Magdalena, "A propósito del rescate de cautivos conforme a las fuentes musulmanas de época nazarí", en Anuario de Historia del Derecho Español 73 (2003) 457-495, y posteriormente por ARCAS CAMPOY, María y JIMÉNEZ ALCÁZAR, Juan Francisco, "Una carta en la frontera de Granada: de la Vera nazarí a la capital de la Gobernación de Orihuela (año 879/1474)", en Estudios de Dialectología Norteafricana y Andalusi 10 (2006) 81-92. 
conserva la respuesta ${ }^{24}$ del Consell de Orihuela acusando recibo y alegando no tener responsabilidad alguna en el caso. Sea como fuere, lo cierto es que los cautiverios siempre fueron un tema muy complejo donde intervenían muchos factores que incidían en perjuicio de las personas que caían en tal situación.

Por último cabe destacar el interés filológico del documento ya que se trata de una carta escrita en árabe en los últimos años del reino nazarí que contiene varios rasgos léxicos, morfológicos y sintácticos del haz dialectal andalusí en su modalidad granadina.

\section{CONCLUSIONES}

De todo lo expuesto se desprende que los documentos oficiales conservados, pese a ser escasos, junto con las referencias a escritos no conservados de carácter legal suponen una estimable contribución al conocimiento de la vida social, política y económica de la frontera nordeste del reino de Granada en el siglo XV. Se trata, pues, de una contribución siempre abierta a la búsqueda y consulta de nuevos documentos que permitan aportar mayor información sobre el tema.

${ }^{24}$ Archivo Histórico de Orihuela, A-32, fol. 25r. 